\title{
Über die Fettsäuren aus grünen Blättern und das Vorkommen der $\triangle^{3}$-trans-Hexadecensäure
}

\author{
Von Hildegard Debuch \\ Aus dem Physiologisch-chemischen Institut der Universität zu Köln, \\ (Direktor: Prof. Dr. Dr. E. KLenK) \\ (Z. Naturforschg. 16 b, 561-567 [1961] ; eingegangen am 12. Mai 1961) \\ Meinem verehrten Lehrer, Herrn Prof. KLENK zum 65. Geburtstag gewidmet
}

\begin{abstract}
Es wurden in Analogie zu früheren ${ }^{1}$ Untersuchungen über die Fettsäuren aus Chloroplasten nun die Fettsäuregemische aus grünen Blättern von Spinat (Spinacia oleracea) und Löwenmaul (Anthirrhinum majus, Sippe 50) dargestellt und mit Hilfe gaschromatographischer Methoden analysiert. Dabei zeigte sich:

1. Das Vorkommen einer $\mathrm{C}_{16}$-Monoensäure (sowohl in den Spinat- als auch in den Löwenmaul-Fettsäuren), die auf Grund von IR-Spektren und oxydativem Ozonidabbau einwandfrei als $\Delta^{3}$-transHexadecensäure anzusprechen ist.

2. Die Fettsäuregemische vorgenannter grüner Blätter unterscheiden sich nur unwesentlich voneinander. Allerdings konnten nur im Spinat bestimmbare Mengen einer Hexadecatriensäure, nur im Löwenmaul solche einer Octadecatetraensäure nachgewiesen werden.

3. Die Untersuchung der UV-Spektren der nicht isomerisierten Säuren verglichen mit isomeri sierten Proben ergibt keinen Anhalt für das Vorhandensein größerer Mengen von konjugierten Polyensäuren.

4. Beim Vergleich der Fettsäuren aus Löwenmaulblättern und Chloroplasten aus Löwenmaul ist ein niedrigerer Gehalt ersterer an Linolensäure zugunsten von Linol- und Palmitinsäure festzustellen. $\mathrm{C}_{20}$-Fettsäuren konnten nur in Blatt- nicht aber in Chloroplasten-Fettsäuren aufgefunden werden.
\end{abstract}

Über die Fettsäuren aus grünen Blättern liegen bisher nur wenige Mitteilungen vor. Untersucht wurden vor allem verschiedene Arten von Futtergräsern, die den Weidetieren als ausschließliche Nahrungsquelle dienen. Durch Bestimmung der FettsäureZusammensetzung des Futters und die der Lipoide verschiedener Tierarten konnten so gewisse Aussagen über den Umbau des Nahrungsfettes gemacht werden. Vergleicht man die Ergebnisse verschiedener Autoren über die Fettsäuren aus Futtergräsern $^{2-5}$, Kohlblättern ${ }^{6}$, Spinat ${ }^{7}$ oder anderen grünen Blättern ${ }^{8,9}$, so fällt stets der hohe Gehalt an $\mathrm{C}_{18}$-Polyensäuren (Linol- und vor allem Linolensäure) auf. Unter den gesättigten Fettsäuren war stets Palmitinsäure neben nur kleinen Mengen von Stearinsäure vorhanden.

Die in pflanzlichen Samenlipoiden und Lipoiden

1 H. Debuch, Z. Naturforschg. 16 b, 246 [1961].

2 J. A. B. Smith u. A. C. Chibnall, Biochem. J. 26, 218 [1932]

3 J. A. B. Smith u. A. C. Chibnall, Biochem. J. 26, 1345 [1932]

4 F. B. Shorland, Nature [London] 153, 168 [1944].

5 G. A. Garton, Nature [London] 187, 511 [1960].

6 A. C. Chibnall u. H. J. Channon, Biochem. J. 21, 479 [1927].

7 J. H. SPeER, E. C. Wise u. M. C. Hart, J. biol. Chemistry 82, 105 [1929].

8 W. M. Crombie, J. exp. Botany 9, 254 [1958]. tierischer Herkunft so weit verbreitete und oft mengenmäßig vorherrschende Ölsäure stellt nur einen kleinen Teil der Blattfettsäuren dar neben einer etwa in gleichem Prozentsatz stets aufgefundenen Hexadecensäure.

Seit der Auffindung der Vaccensäure, die von ihrem Entdecker Bertram ${ }^{10}$ als $\Delta^{11}$-trans-Octadecensäure angesprochen und von ihm als Bestandteil des Rinder-, Schaf- und Butterfettes als erste in der Natur aufgefundene Trans-Fettsäure beschrieben wurde, konnte sie häufig vor allem im Fett von Wiederkäuern ${ }^{11-14}$ aufgefunden werden. Durch Unterschiede der IR- und Röntgenspektren von synthetischer $\Delta^{11}$-Octadecensäure (trans) und natürlicher „Vaccensäure" suchte man später nach weiteren Isomeren. So konnten Hilditch und Mitarbb. ${ }^{15}$ neben der $\Delta^{11}$ - auch $\Delta^{10}$-Octadecensäure (trans) im

9 F. B. Shorland, Nature [London] 156, 269 [1945].

10 S. H. Bertram, Biochem. Z. 197, 433 [1928].

11 J. Grossfeld u. A. Simmer, Z. Unters. Lebensmittel 59, 237 [1930].

12 P. C. Rao u. B. F. Daubert, J. Amer. chem. Soc. 70, 1102 [1948].

13 D. G. Cornwell, R. Backderf, C. L. Wilson u. J. B. Brown, Arch. Biochem. Biophysics 46, 364 [1953].

14 L. Hartmann, F. B. Shorland u. I. R. C. McDonald, Biochem. J. 61, 603 [1955].

15 S. S. Gupta, T. P. Hilditch, S. Paul u. R. K. Shrivastava, J. chem. Soc. [London] 1950, 3484. 
Körper- und Milchfett von Rindern und Schafen nachweisen.

In bezug auf die Entstehung dieser Trans-Fettsäure wurde von SwERn und Mitarbb. ${ }^{16}$ zunächst angenommen, daß es bei evtl. Wanderung der Doppelbindung durch Einwirkung von Oxydasen zu einer Cis-Trans-Umlagerung bei Wiederkäuern kommt, die dann in ihrem Depotfett die Trans-Fettsäuren ablagern. Oxydasen wurden jedoch auch in Nicht-Wiederkäuern gefunden, bei denen Trans-Fettsäuren nur spurenweise vorkommen. Deshalb suchte man in der Nahrung der Weidetiere nach Trans-Fettsäuren. Sie wurden unseres Wissens erst durch Shorland und Mitarbb. ${ }^{14}$ in Futtergräsern in Mengen von 0,6 bis $2,2 \%$ der Gesamtfettsäuren aufgefunden. Diese wurden nicht auf ihre Kettenlänge hin untersucht. Da jedoch vorgenannte Autoren annehmen, daß der überwiegende Teil der Trans-Fettsäuren durch Bakterieneinwirkung im Pansen der Wiederkäuer entsteht, wobei es zur teilweisen Hydrierung vor allem der Linolensäure der Nahrung und damit sowohl zu Cis-Trans-Isomerisierung als auch zur Umlagerung der Doppelbindung kommen kann, ist anzunehmen, daß die Autoren in den Futtergräsern keine transHexadecensäure vermuten.

Auch $\mathrm{GARTON}^{5}$ fand bei der gaschromatographischen Untersuchung der Fettsäuren eines Gemisches von Futtergräsern eine Hexadecensäure, die er als Palmitoleinsäure ${ }^{5,17}$ ( $\Delta^{9}$-cis-Hexadecensäure $)$ bezeichnete.

Um so auffallender ist das Auftreten einer Hexadecensäure im Fettsäuregemisch von Spinat- und Löwenmaulblättern *, die sich als identisch mit einer erst kürzlich ${ }^{18}$ in der Algenart Scenedesmus erstmals aufgefundenen Säure erwies. Sie kommt zwar nur in kleinen Mengen in den hier untersuchten Blättern vor, (Spinat 2,6\%; Löwenmaul 1,3\% der Gesamtfettsäuren) konnte jedoch mit Sicherheit als $\Delta^{3}$-transHexadecensäure erkannt werden. Im Gaschromatogramm der Gesamtfettsäuren aus Spinat und Löwenmaul (s. Abb. 3) fiel zunächst ein Maximum kurz hinter der Palmitinsäurezacke auf, das sich von letzterer deutlicher abgrenzte als die der Palmitoleinsäure. Durch Zugabe von jeweils Palmitin-, Palmitolein- oder einer $\mathrm{C}_{16}$-Diensäure $\mathrm{zu}$ der zu untersuchenden Substanz, die sich durch fraktionierte Tief-

\footnotetext{
16 D. Swern, H. B. Knight u. C. R. Eddy, J. Amer. Oil Chemists' Soc. 29, 44 [1952].

17 G. A. Garton, Nutrit. Abstr. a. Rev. 30, 1 [1960].
}

kühlkristallisation anreichern ließ (s. Abb. $5 \mathrm{a}-\mathrm{c}$ ), wurde eine trans- $\mathrm{C}_{16}$-Monoensäure vermutet (s. Abb. 1).

Wegen der geringen Substanzmenge ließ sich diese Säure zwar nicht rein isolieren, jedoch konnte eine Fraktion erhalten werden, die praktisch nur aus Palmitin- und der gesuchten Säure bestand (s. Abb. 5 c). Diese Fraktion zeigte im IR-Spektrum eine deutliche Absorption bei $965 \mathrm{~cm}^{-1}$, was für das Vorhandensein einer Doppelbindung mit trans-Konfiguration spricht (s. Abb. 6).

Nach oxydativem Ozonidabbau konnte als einzige Monocarbonsäure neben der in dem Ausgangsgemisch vorhandenen Palmitinsäure nur Tridecansäure (s. Abb. 7), als einzige Dicarbonsäure nur Malonsäure (s. Abb. 8) nachgewiesen werden. Aus diesen Ergebnissen muß geschlossen werden, daß der $\mathrm{C}_{16}$-Monoensäure die $\Delta^{3}$-trans-Konfiguration zukommt. Unseres Wissens wurde diese Fettsäure hiermit erstmals in grünen Blättern aufgefunden. Über ihre Bedeutung kann nichts ausgesagt werden, jedoch scheint es sich bei der Hexadecensäure in Chloroplasten-Fettsäuren ${ }^{1}$ ebenfalls um die $\Delta^{3}$-trans-Hexadecensäure zu handeln, da sie im Gaschromatogramm das gleiche Retentionsvolumen besitzt.

Was die Zusammensetzung der hier untersuchten Blattfettsäuren anbetrifft, so lassen sich keine wesentlichen Unterschiede hervorheben (s. Tab. 1).

Im Ganzen ist eher eine auffallende Übereinstimmung der beiden Fettsäuregemische festzustellen. Lediglich das Auftreten der $\mathrm{C}_{16}$-Trien- und der $\mathrm{C}_{18}$-Tetraensäure war in beiden Blattsorten deutlich verschieden. (Erstere ist in Rapsblättern zu etwa $10 \%$ der Gesamtfettsäuren vorhanden ${ }^{9}$.) Die Menge der Ölsäure im Spinat übertrifft diejenige im Löwenmaul um das Dreifache. Allerdings können die Befunde von SpeEr und Mitarbb. ${ }^{7}$ nicht bestätigt werden, nach denen $26,3 \%$ der Gesamtfettsäuren der Triglyceride aus Spinat aus Ölsäure bestehen sollen. Es muß jedoch erwähnt werden, daß in der vorliegenden Arbeit die Gesamtfettsäuren erfaßt wurden.

Bei der Gegenüberstellung der Blatt- und Chloroplasten-Fettsäuren von Antirrhinum majus zeigt sich, daß letztere einen größeren Prozentsatz von Polyensäuren, vor allem Linolensäure besitzen. Ob das Fehlen der Arachinsäure ein typisches Chloroplasten-

\footnotetext{
* Für die Überlassung der Pflanzen danke ich Herrn Prof. Dr. W. MEnKE aufs herzlichste sowie für sein Interesse an der Arbeit.

18 W. Knipprath, Dissertation, Köln 1961.
} 

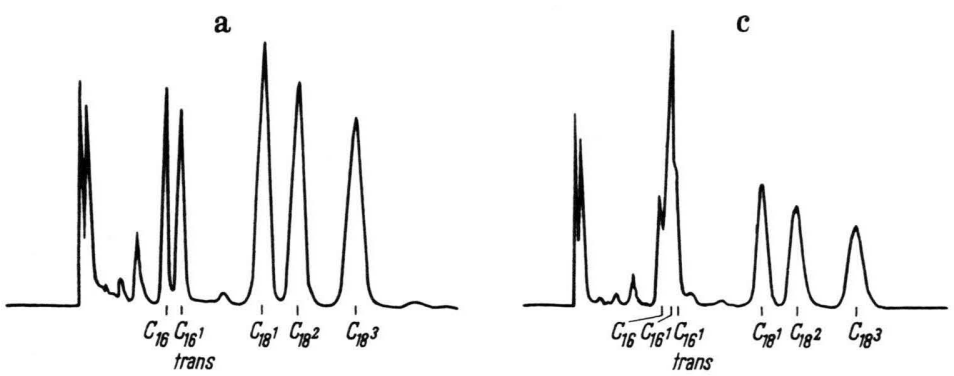

Abb. 1. Gaschromatographische Untersuchung der $\mathrm{C}_{16}$-Monoensäure aus Spinat. Argon Chromatograph W. G. Pye und Co. Ltd. Granta Works, Cambridge, England. Ionisationsdetektor, Säule $123 \mathrm{~cm}$, Durchmesser $4 \mathrm{~mm}$, Stationäre Phase Reoplex 400, Trägermaterial: Celite (Perkin Elmer Präparat 098-1504 gewaschen und gesiebt). T. Säule: $181{ }^{\circ} \mathrm{C}$; Zelle: $181^{\circ} \mathrm{C}$; Verdampfer: $273^{\circ} \mathrm{C}$. a) Fettsäurefraktion A der destillierten Fettsäuremethylester aus Spinatblättern (s. Abb. 4). b) Substanz A +Palmitinsäure. c) Substanz A+Palmitoleinsäure. d) Substanz A + Palmitinsäure $+\mathrm{C}_{16}$-Diensäure.

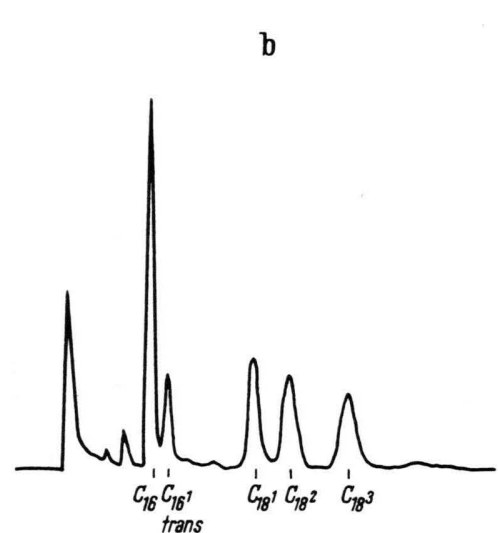

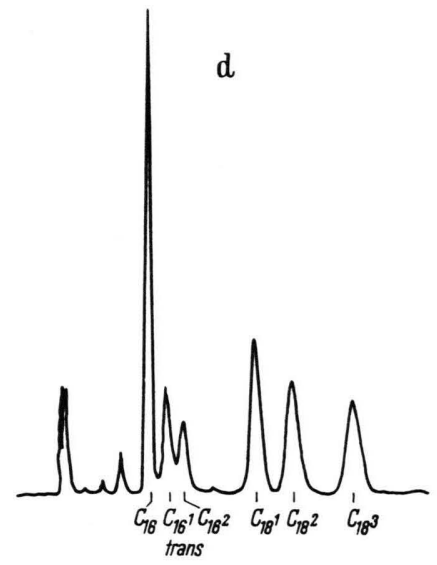

merkmal ist, kann z. Zt. noch nicht entschieden werden.

ZIRM und Mitarbb. ${ }^{19}$ teilten 1955 mit, daß sie in verschiedenen grünen Blättern u. a. auch in SpinatBlättern „große Mengen“ konjugierter Fettsäuren durch Untersuchung der UV-Spektren der nicht isomerisierten Säuren fanden. Wir führten eine Alkali- isomerisierung mit der bei $-70{ }^{\circ} \mathrm{C}$ in Aceton gelösten Fraktion (s. Abb. 4) nach fraktionierter Tiefkühlkristallisation der destillierten Fettsäuren sowohl von Spinat als auch von Löwenmaul durch und nahmen unter gleichen Bedingungen das UV-Spektrum einer nicht isomerisierten Probe auf (s. Abb. 2).

\begin{tabular}{|c|c|c|c|c|c|c|c|c|c|c|}
\hline \multirow[b]{2}{*}{$\begin{array}{l}\text { Ausgangs- } \\
\text { substanz }\end{array}$} & \multirow{2}{*}{$\begin{array}{l}\mathrm{C}_{14} \\
\text { Myristin- } \\
\text { sre. }\end{array}$} & \multicolumn{3}{|c|}{$\mathrm{C}_{16}$} & \multicolumn{5}{|c|}{$\mathrm{C}_{18}$} & \multirow{2}{*}{$\begin{array}{c}\mathrm{C}_{20} \\
\begin{array}{c}\text { Arachin } \\
\text { sre. }\end{array}\end{array}$} \\
\hline & & $\begin{array}{l}\text { Palmitin- } \\
\text { sre. }\end{array}$ & $\begin{array}{l}\Delta^{3} \text {-trans- } \\
\text { Monoen- } \\
\text { sre. }\end{array}$ & $\begin{array}{l}\text { Trien- } \\
\text { sre. }\end{array}$ & $\begin{array}{l}\text { Stearin- } \\
\text { sre. }\end{array}$ & $\begin{array}{l}\text { Öl- } \\
\text { sre. }\end{array}$ & $\begin{array}{l}\text { Linol- } \\
\text { sre. }\end{array}$ & $\begin{array}{l}\text { Linolen- } \\
\text { sre. }\end{array}$ & $\begin{array}{l}\text { Tetraen- } \\
\text { sre. }\end{array}$ & \\
\hline $\begin{array}{l}\text { Spinacia } \\
\text { oleracea (Blatt) }\end{array}$ & 0,2 & 12,9 & 2,6 & 4,6 & Spur & 6,6 & 16,3 & 56,2 & Spur & 0,6 \\
\hline $\begin{array}{l}\text { Antirrhinum } \\
\text { majus (Blatt) }\end{array}$ & 0,1 & 13,4 & 1,3 & Spur & 2,4 & 1,8 & 17,7 & 57,9 & 3,6 & 1,8 \\
\hline $\begin{array}{l}\text { Antirrhinum } \\
\text { majus } \\
\text { (Chloroplasten) }\end{array}$ & Spur & 9,5 & 1,3 & 0,2 & 1,0 & 1,6 & 15,1 & 71,3 & - & - \\
\hline
\end{tabular}

Tab. 1. Zusammensetzung der destillierten Fettsäuremethylester (in \% der Gesamtfettsäuren) .

19 K. J. Zirm, A. Pongratz u. W. Polesofsky, Biochem. Z. 326, 405 [1955]. 


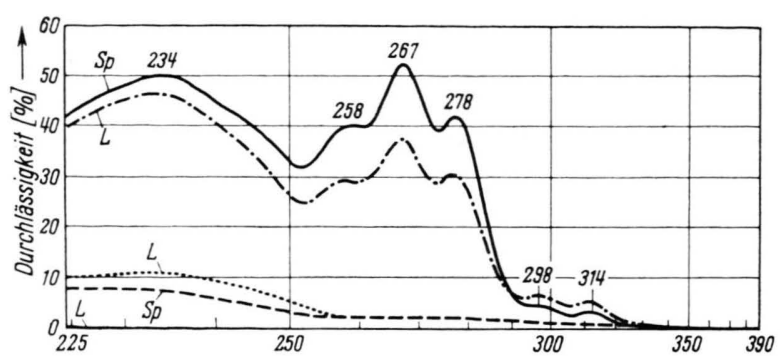

Abb. 2. UV-Spektrum der —_, - - - - isomerisierten und ........ nicht isomerisierten hochungesättigten Fettsäurefraktionen aus Spinatblättern (Sp) und Löwenmaulblättern (L) (1-proz. Lösg., $d=1 \mathrm{~cm}$ ).

Wie aus Abb. 2 zu ersehen ist, kann es sich nur um kleine Mengen konjugierter Fettsäuren handeln, die möglicherweise bei der Aufarbeitung (2-maliges Verseifen und Verestern und Destillieren!) gebildet wurden. Damit dürften die Befunde von Crombie ${ }^{8}$ bestätigt werden, die konjugierte $\mathrm{C}_{18}$-Polyensäuren in grünem und weißem Blattgewebe verschiedener Arten nur spurenweise finden konnte.

\section{Beschreibung der Versuche}

\section{Darstellung der Fettsäuren*}

Die frisch geernteten grünen Blätter aus Spinat und Löwenmaul wurden bei etwa $4{ }^{\circ} \mathrm{C}$ durch eine 3 -Walzenmühle gemahlen und gefriergetrocknet.
$798,6 \mathrm{~g}(905,4 \mathrm{~g})$ des getr. Materials dreimal jeweils mit der $1^{1 / 2}$-fachen Menge Chloroform : Methanol (1:3 V/V) I Stde. unter Rückfluß extrahiert und abgesaugt. Die vereinigten Extrakte i. V. eingeengt, Rückstand in Äther aufgenommen. Ätherunlösliches abzentrifugiert.

Ätherlösung (dunkelgrün gefärbt) i. V. eingeengt und diesen Rohlipoidextrakt sofort $1^{1 / 2}$ Stdn. mit der 15fachen Menge 4-proz. trockener methanolischer $\mathrm{HCl}$ unter Rückfluß gespalten. Lösung wiederholte Male mit Petroläther (bis $56^{\circ}$ siedend) ausgeschüttelt, die vereinigten Petrolätherextrakte neutral gewaschen, über $\mathrm{Na}_{2} \mathrm{SO}_{4}$ getrocknet, abdestilliert.

Fettsäuremethylester und Unverseifbares: $47,0 \mathrm{~g}$ $(41,0 \mathrm{~g})$; entsprechend $5,9 \%(4,5 \%)$ der Ausgangssubstanz. Verseifung: 1 Stde. mit der 30-fachen Menge 1/2-n. methanol. $\mathrm{NaOH}$ unter Rückfluß. Nach Stehen über Nacht bei $0^{\circ} \mathrm{C}$ feste Seifen abgesaugt, 3-mal in Petroläther suspendiert, jeweils abgesaugt. Die flüssigen Seifen 3-mal mit Petroläther gewaschen. Vereinigte Petrolätherextrakte zunächst mit 50-proz. Methanol, schließlich mit Wasser seifenfrei gewaschen, dann ge trocknet. Rückstand: Unverseifbares $11,0 \mathrm{~g}(10,8 \mathrm{~g})$.

Feste und flüssige Seifen einschließlich WaschMethanol und -Wasser mit verdünnter $\mathrm{HCl}$ angesäuert und mit Petroläther-Äther $(1: 1 \mathrm{~V} / \mathrm{V})$ extrahiert. Vereinigte Ätherextrakte nach Trocknen über $\mathrm{Na}_{2} \mathrm{SO}_{4}$ eingeengt. Rückstand: Fettsäuren: $31,4 \mathrm{~g}(27,0 \mathrm{~g})$.

Reinigung der dunkelbraun-grün gefärbten Fettsäuren: Diese wieder mit methanol. $\mathrm{HCl}$ in die Ester überführt. $30,6 \mathrm{~g}(25,6 \mathrm{~g})$ davon ohne Fraktionierung im Hochvakuum bei $10^{-4}$ Torr schnell überdestilliert. Destillat nur schwach gelb gefärbt: $25,6 \mathrm{~g}(22,1 \mathrm{~g})$. Gaschromatographische Untersuchung des Fettsäuregemisches (s. Abb. 3).
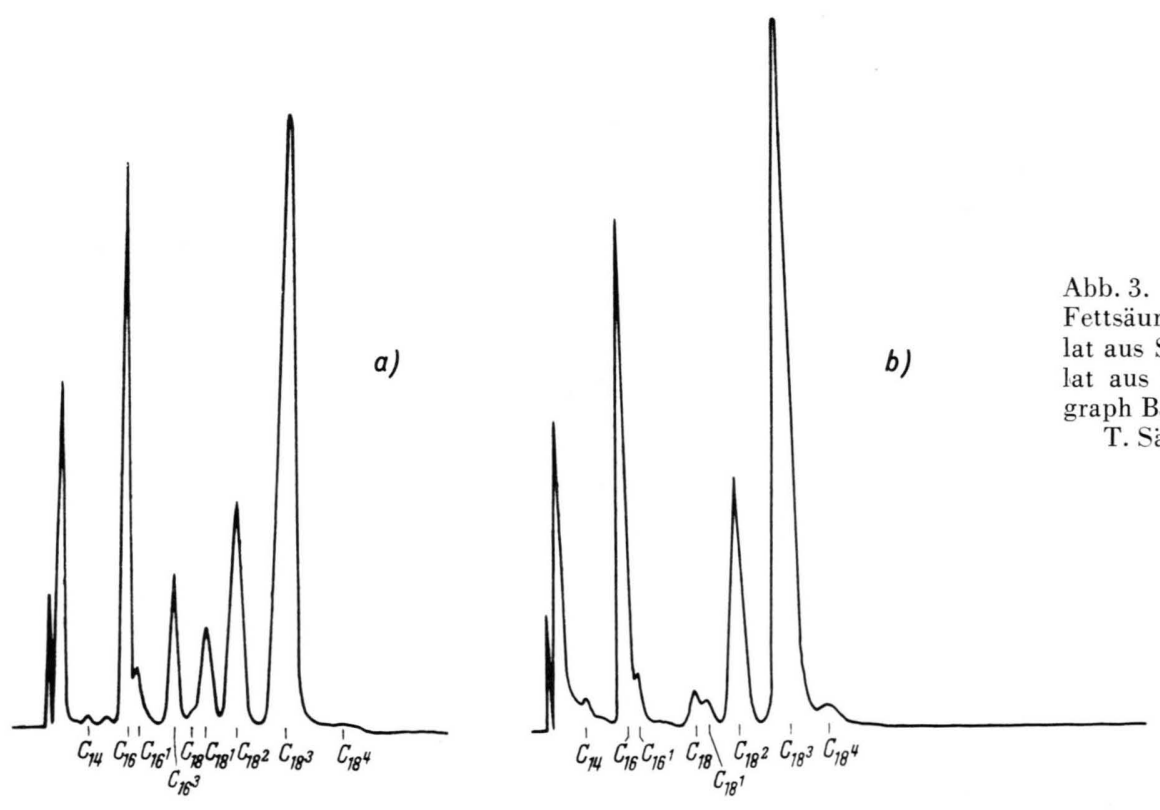

* Die in Klammern befindlichen Zahlen gelten für Löwenmaul. 
Zur Berechnung der Zusammensetzung, die auf die bereits früher ${ }^{1}$ beschriebene Weise erfolgte, wurde jeweils eine Probe hydriert (s. Tab. 1).

Die Berechnung der hydrierten Gemische ergab: $\mathrm{C}_{14}: 0,2 \%(0,1 \%) ; \mathrm{C}_{16}: 20,1 \%(14,7 \%) ; \mathrm{C}_{18}: 79,1 \%$ $(83,4 \%) ; \mathrm{C}_{29}: 0,6 \%(1,8 \%)$.

\section{Tiefkühlkristallisation *}

Methylester verseift. Mit 22,0 g (18,7 g) der freien Fettsäuren Tiefkühlkristallisation nach SHINowara und Brown ${ }^{20}$ durchgeführt: Substanz jeweils in der 15fachen Menge trockenen Acetons gelöst, sehr langsam in Trockeneis-Aceton abgekühlt. Nach 2-stdg. Stehen bei der gewünschten Temperatur, Kristalle mit Umkehrnutsche abgetrennt, kurz i. V. getrocknet, erneut in der 15-fachen Menge Aceton gelöst und behandelt wie vorher.

Aceton-Lösungen vereinigt, eingeengt, Rückstand behandelt wie oben (s. Abb. 4).

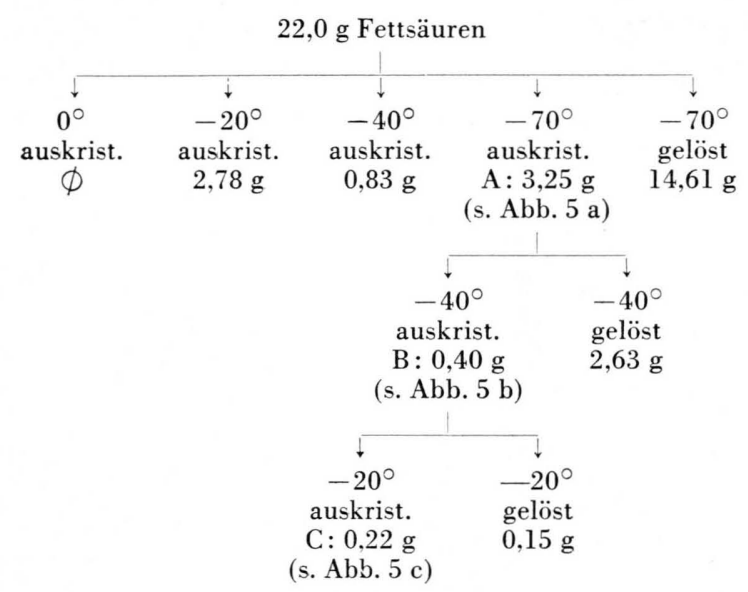

Abb. 4. Übersicht über die Tiefkühlkristallisation der Fettsäuren aus Spinat.

Von jeder der gewonnenen Fettsäurefraktionen wurde eine kleine Menge auf die übliche Weise verestert und gaschromatographisch untersucht.

Wie aus Abb. $5 \mathrm{a}-\mathrm{c}$ zu ersehen ist, gelang es durch fraktionierte Tiefkühlkristallisation ein Fettsäuregemisch zu gewinnen, das praktisch nur aus Palmitin- und der $\mathrm{C}_{16}$-Monoensäure bestand.

Daß es sich dabei um das Trans-Isomere handelt, ist aus der Absorption bei $965 \mathrm{~cm}^{-1}$ im IR-Spek-

* Der Einfachheit halber wird diese nur für die Fettsäuren aus Spinat beschrieben. Die Tiefkühlkristallisation für d:o Löwenmaul-Fettsäuren wurde analog durchgeführt. trum (Abb. 6) zu ersehen. (Das IR-Spektrum der entsprechenden Fettsäurefraktion aus Löwenmaul zeigte die gleiche Absorptionsbande.)
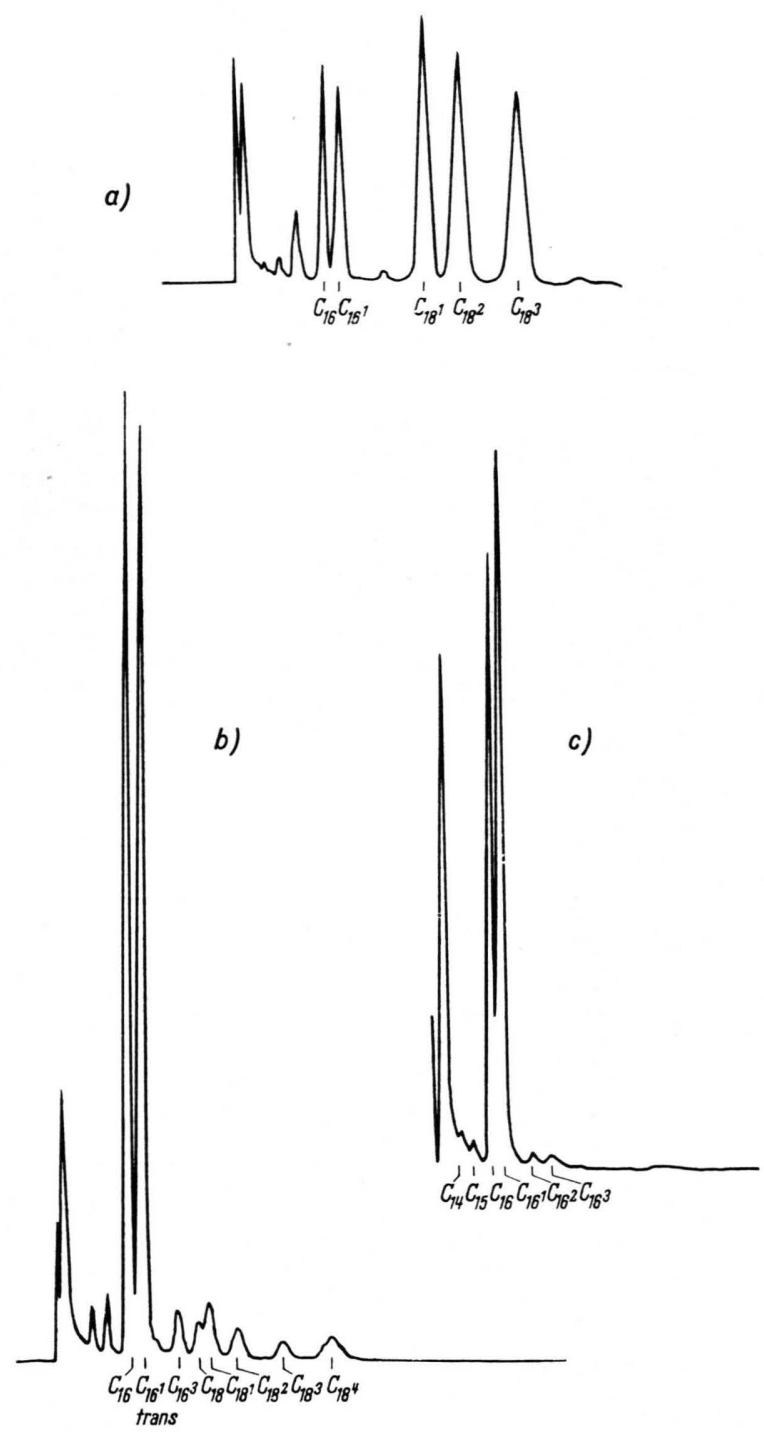

Abb. 5. Gaschromatographische Untersuchung der durch fraktionierte Tiefkühlkristallisation gewonnenen Fettsäure-Fraktionen (s. Abb. 4) aus Spinat. a) Substanz A (s. Abb. 4). Chromatograph: Pye (näheres s. Abb. 1) T. Säule: $181^{\circ} \mathrm{C}$; Zelle: $181^{\circ} \mathrm{C}$; Verdampfer: $273^{\circ} \mathrm{C}$. b) Substanz B (s. Abb. 4) Chromatograph: Pye s. Abb. 1. T. Säule: $182{ }^{\circ} \mathrm{C}$; Zelle: $182{ }^{\circ} \mathrm{C}$; Verdampfer: $266^{\circ} \mathrm{C}$. c) Substanz C (s. Abb. 4). Chromatograph: Barber-Colman (näheres s. l. c. ${ }^{1}$ ) T. Säule $190^{\circ} \mathrm{C}$; Zelle: $231^{\circ} \mathrm{C}$; Verdampfer : $289^{\circ} \mathrm{C}$.

20 G. Y. Shinowara u. J. B. Brown, J. biol. Chemistry 134 , 331 [1940]. 


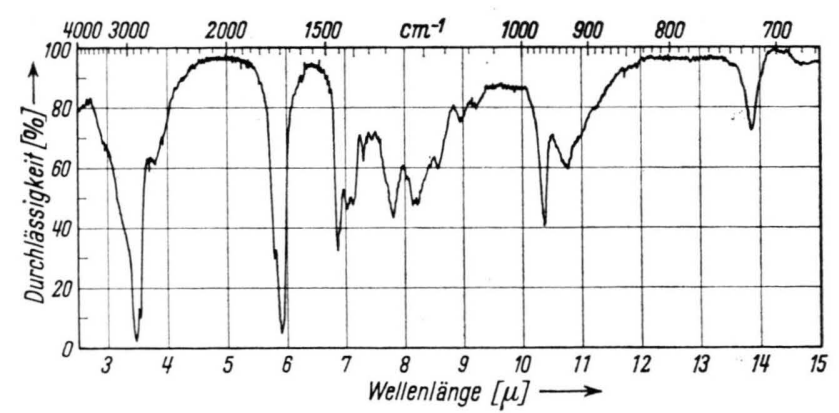

Abb. 6. IR-Spektrum der Fettsäure-Fraktion C aus Spinat (s. Abb. 4 und Abb. 5 c).

\section{I s o m erisierung}

Zur Prüfung auf Konjuensäuren wurde $1 \mathrm{mg}$ der bei $-70^{\circ}$ in Aceton in Lösung befindlichen, vom Lösungsmittel befreiten Fettsäurefraktion in $0,5 \mathrm{ml}$ 23-proz. KOH-Glykol $8 \mathrm{Min}$. bei $180^{\circ} \mathrm{C}$ isomerisiert ${ }^{21}$ und das Spektrum aufgenommen ${ }^{22}$. Zum Vergleich wurde von einer 1-proz. Lösung der gleichen Fettsäure-Fraktion in Methanol das UV-Spektrum aufgenommen (s. Abb. 2).

4. Ozonisierung und oxydative Spaltung der Trans-Monoensäure-Fraktion ( $\mathrm{n}$ a $\mathrm{ch}$ KLenk und Bongard $)^{23}$

$58,81 \mathrm{mg}(52,6 \mathrm{mg})$ der Fettsäure-Fraktion $\mathrm{C}$ aus Spinat (und der entsprechenden aus Löwenmaul) in $8 \mathrm{ml}$ Eisessig: Methylacetat (1 : 1 V/V) gelöst. Bei Zim-
mer-Temperatur ozonisiert. Indikator: Sudanrot. Weitere Oxydation: Ozonidlösung mit $10 \mathrm{ml}$ Eisessig in Schliffkölbchen überführt, mit 1,5 ml Perhydrol versetzt und 3 Tage bei $36{ }^{\circ} \mathrm{C}$ stehen gelassen. Um die entstandene Peressigsäure zu zerstören, wurde kurz aufgekocht. Lösungsmittel bei $40^{\circ} \mathrm{C}$ über eine Kolonne $(40 \mathrm{~cm})$ i. V. restlos entfernt. Rückstand mit $7 \mathrm{ml}$ Petroläther versetzt, 2 Stdn. bei $0^{\circ}$ stehen gelassen. Die Dicarbonsäuren kristallisierten aus. Petroläther abdekantiert, mit Wasser gewaschen, über $\mathrm{Na}_{2} \mathrm{SO}_{4}$ getrocknet und abdestilliert.

Rückstand: Monocarbonsäuren: 53,1 mg (40,5 $\mathrm{mg}$ ), die zur gaschromatographischen Untersuchung verestert wurden.

Wie aus Abb. 7 zu ersehen ist, handelt es sich um ein Gemisch von Palmitinsäure und Tridecansäure.
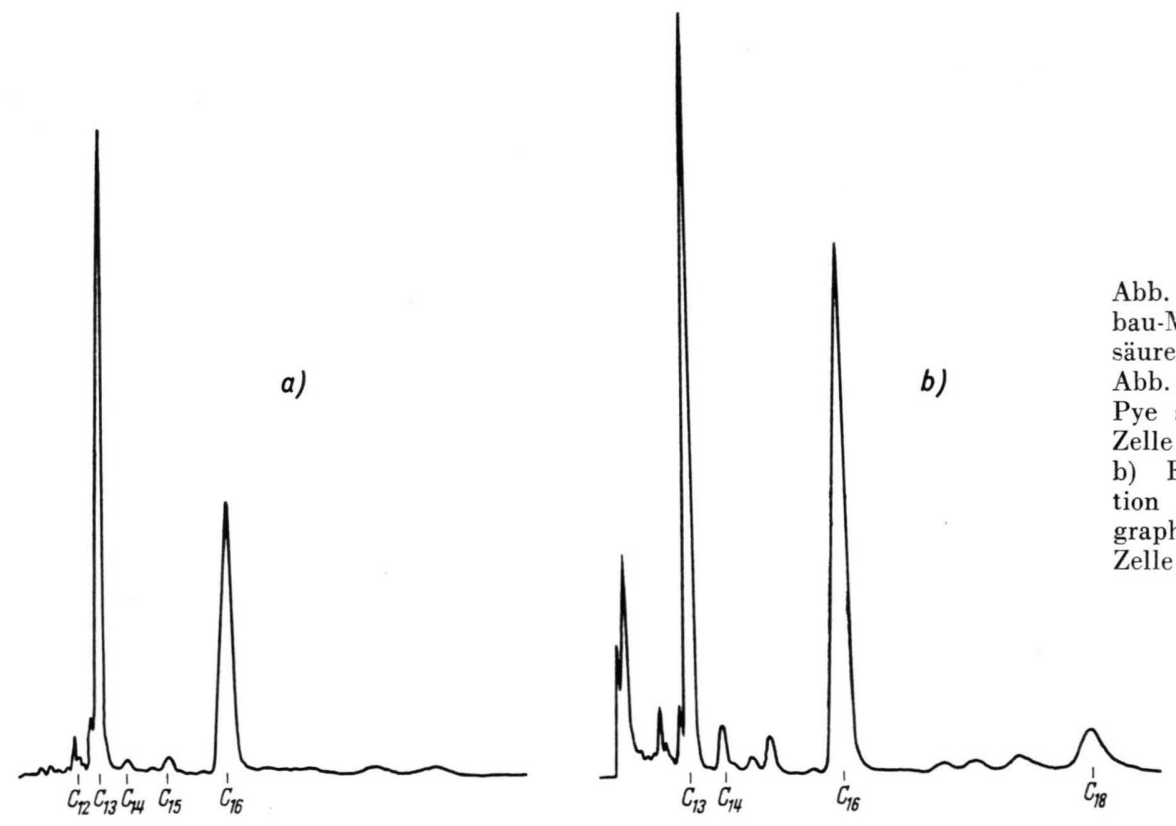

21 R. T. Holman u. G. O. Burr, Arch. Biochemistry 19, 474 [1948].

22 E. Klenk u. F. Lindlar, Hoppe-Seyler's Z. Physiol. Chem.

23 E. Klenk u. W. Bongard, Hoppe-Seyler's Z. physiol. Chem. 290, 181 [1952]. 299, 74 [1954]. 
Letztere war aus der $\mathrm{C}_{16}$-Monoensäure durch oxydativen Abbau entstanden.

Die im Kölbchen befindlichen Kristalle wurden im Waschwasser gelöst und gefriergetrocknet. Rückstand: Abbaudicarbonsäuren: 17,7 mg (11,2 mg). Wegen der kleinen Menge wurden diese lediglich papierchromatographisch untersucht (nach KLENK und TomUSCHAT ${ }^{24}$ ).

Absteigende Chromatographie: (Schleicher \& Schüll 2043 b) Mobile Phase: untere Phase eines Gemisches aus $10 \mathrm{Tln}$. Eisessig, $100 \mathrm{Tln}$. eines 5-proz. Butanol enthaltenden Chloroforms und $100 \mathrm{Tln}$. Wasser, das bei $25-28{ }^{\circ} \mathrm{C} 1$ Stde. äquilibriert wurde. Stabile Phase: obere Phase des beschriebenen Gemisches. Jeweils $50 \gamma$ Fettsäure aufgetragen. Laufzeit $5-6 \mathrm{Stdn} ., T_{.}: 25^{\circ} \mathrm{C}$. Vor der Anfärbung wurde geprüft, ob das Papier völlig frei von Essigsäure war. Nachweis der Dicarbonsäuren: 1 Teil 1-n. KJ; 1 Teil $0,2-n . \mathrm{KJO}_{3} ; 20$ Tle. 3-proz. Stärke; 20 Tle. Wasser. (Lösung kurz vor Gebrauch ansetzen. Falls sie blau gefärbt ist, muß mit $\mathrm{Na}_{2} \mathrm{~S}_{2} \mathrm{O}_{3}$ $(n / 10)$ bis zur Farblosigkeit titriert werden). Das Chromatogramm zeigte nach dem Einsprühen (5 bis 10 Min.) violette Flecken auf weißem Grund.

24 E. KlenK u. H. J. Tomuschat, noch unveröffentlicht.

Abb. 8. Papierchromatogramm der Abbau-Dicarbonsäuren der Fettsäure-Fraktion $\mathrm{C}$ aus Spinat (nach KLenK und TomuSCHAt ${ }^{24}$ ). 1. Abbau-Dicarbonsäuren der Fettsäure-Fraktion, 2 Test: Pimelinsäure, 3 Test: Adipinsäure, 4 Test: Glutarsäure, 5 Test: Bernsteinsäure, 6 Test: Malonsäure.

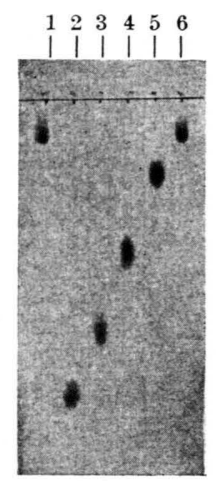

Wie aus Abb. 8 zu ersehen ist, waren als AbbauDicarbonsäuren nur Malonsäure nachweisbar.

Die Abbau-Dicarbonsäuren der entsprechenden Fettsäure-Fraktion aus Löwenmaulblättern ergaben das gleiche Bild.

Die Arbeit wurde mit Unterstützung der De u t $\mathrm{schen}$ Forschungsgemeinschaf turchgeführt. Frl. D. BIELEFELd sei für ihre Mitarbeit gedankt.

\title{
Über die Phosphatkapazität der Gärung und ihr Modell als Ursachen des Crabtree-Effekts der Krebszellen
}

(Mit einer experimentellen Entgegnung auf eine Arbeit von

K. Letnansky und F. Seelich ${ }^{1}$ )

Von Lutz Kiesow

Aus dem Physiologisch-Chemischen Institut der Freien Universität Berlin, Berlin-Dahlem

(Direktor: Prof. Dr. Dr. ERNSt Schütte)

(Z. Naturforschg. 16 b, 567-575 [1961] ; eingegangen am 5. Juni 1961)

\begin{abstract}
Da Glucose und 2-Desoxy-D-glucose in Ascites-Krebszellen gleich große Erniedrigungen der Orthophosphat-Konzentration verursachen, hemmen sie die Atmung dieser Zellen gleich stark. Sie führen beide zu gleich großem C r a b t r e e - Effekt.
\end{abstract}

"The tentative conclusion is that the glycolytic activity exerts a significant chocking effekt on the capacity for respiration of tumour tissue." Mit diesem Satz faßte Crabtree ${ }^{2} 1929$ seine Experimente zusammen, die zu dem Ergebnis führten, das wir heute als $\mathrm{C}$ r a b t r e e - Effekt bezeichnen. Im Gegensatz zur P a s t e u r - Reaktion, die die normalen, die embryonalen und die Krebszellen besitzen - die letzteren zeigen sie sogar, genau so wie die wachsen-

1 K. Letnansky u. F. Seelich, Z. Krebsforsch. 64, 1 [1960].

2 H. G. Crabtree, Biochem. J. 23, 536 [1929].

3 L. Kiesow, Z. Naturforschg. 14 b, 224 [1959]. den Kulturhefezellen ${ }^{3}$, am ausgeprägtesten ${ }^{4}$-, kann man den Crabtree-Effekt nur bei Zellen beobachten, die aerob gären. Man findet ihn also z. B. bei den Ehrlichschen Ascites-Krebszellen und bei Zellen aus der Gewebekultur, aber nicht bei trypsinierten Leber-, Nieren- und Embryonalzellen ${ }^{5}$, wenn man unter physiologischen Bedingungen mißt. Diese Ergebnisse weisen darauf hin, daß man den $\mathrm{Cr}$ a b tree-Effekt nicht als umgekehrten $\mathrm{P}$ a s t e ur-

4 O. Warburg, K. Gawehn u. A.W. Geissler, Z. Naturforschg. $11 \mathrm{~b}, 657$ [1956].

5 L. Kiesow, W. Kattner u. U.Schmitz, Z. Naturforschg. 15 b, 487 [1960]. 